\title{
NUT midline carcinoma of the larynx: an international series and review of the literature*
}

Henrik Hellquist, ${ }^{1}$ Christopher A French, ${ }^{2}$ Justin A Bishop, ${ }^{3}$ Andrés Coca-Pelaz, ${ }^{4}$ Evan J Propst, ${ }^{5}$ António Paiva Correia, ${ }^{6}$ Bo-Yee Ngan, ${ }^{7}$ Ronald Grant, ${ }^{8}$ Nicole A Cipriani, ${ }^{9}$ David Vokes, ${ }^{10}$ Rui Henrique, ${ }^{11}$ Fernando Pardal, ${ }^{12}$ Jose Ramon Vizcaino, ${ }^{13}$ Alessandra Rinaldo ${ }^{14}$ \& Alfio Ferlito ${ }^{14}$

${ }^{1}$ Department of Biomedical Sciences and Medicine, University of Algarve, Faro, Portugal, ${ }^{2}$ Department of Pathology, Brigham and Women's Hospital, Boston, MA, ${ }^{3}$ Departments of Pathology and Otolaryngology - Head and Neck Surgery, Johns Hopkins Medical Institutions, Baltimore, MD, USA, ${ }^{4}$ Department of Otolaryngology, Hospital Universitario Central de Asturias, Oviedo, Spain, ${ }^{5}$ Department of Otolaryngology - Head and Neck Surgery, Hospital for Sick Children, University of Toronto, Toronto, Canada, ${ }^{6}$ University Hospital of South Manchester NHS Foundation Trust, Manchester, UK, ${ }^{7}$ Division of Pathology, Department of Pediatric Laboratory Medicine, Hospital for Sick Children, University of Toronto, ${ }^{8}$ Division of Hematology/Oncology, Department of Pediatrics, Hospital for Sick Children, Toronto, Canada, ${ }^{9}$ Department of Pathology, The University of Chicago Medicine and Biological Sciences, Chicago, IL, USA, ${ }^{10}$ Department of Otolaryngology - Head and Neck Surgery, Auckland City Hospital and Department of Surgery, University of Auckland, Auckland, New Zealand, ${ }^{11}$ Department of Pathology, Portuguese Institute of Oncology, Porto, ${ }^{12}$ Anatomic Pathology Department, Braga Hospital, Braga, ${ }^{13}$ Anatomic Pathology Department, St Antonio General Hospital, Porto Hospitals Centre, Porto, Portugal, and ${ }^{14}$ University of Udine School of Medicine, Udine, Italy

Date of submission 23 October 2016

Accepted for publication 2 December 2016

Published online Article Accepted 7 December 2016

Hellquist H, French C A, Bishop J A, Coca-Pelaz A, Propst E J, Paiva Correia A, Ngan B-Y, Grant R, Cipriani N

A, Vokes D, Henrique R, Pardal F, Vizcaino J R, Rinaldo A \& Ferlito A

(2017) Histopathology 70, 861-868. DOI: 10.1111/his.13143

\section{NUT midline carcinoma of the larynx: an international series and review of the literature}

Aims: NUT midline carcinoma (NMC) is a rare undifferentiated and aggressive carcinoma that locates characteristically to the midline of the head and neck, and mediastinum. NMC is characterized by chromosomal rearrangements of the gene NUT, at $15 \mathrm{q} 14$. The BRD4 gene on $19 \mathrm{q} 13$ is the most common translocation partner forming a fusion oncogene, BRD4-NUT. By the end of 2014, the International NUT Midline Carcinoma Registry had 48 patients treated for NMC. Laryngeal NMC are exceedingly rare, and we report a case series of seven cases.

Methods and results: We searched for cases in files of different hospitals as well as a thorough search of the English language literature. The diagnosis of NMC is made by demonstration of NUT rearrangement either by immunohistochemistry, fluorescence in-situ hybridization (FISH) or reverse transcription-polymerase chain reaction (RT-PCR). We found three previously published cases, and in this series add four cases of our own.

Conclusions: NMC consists of monomorphic, often discohesive, cells with an epithelioid appearance and distinct nucleoli. The tumours typically show abrupt squamous differentiation. The mean age of the patients was 34 years, hence significantly lower than that for conventional laryngeal carcinoma. All tumours were located in the supraglottis and five patients died of the disease after $3,7,8,9$ and

\footnotetext{
Address for correspondence: H Hellquist, Department of Biomedical Sciences and Medicine, Campus de Gambelas, University of Algarve, 8005-139 Faro, Portugal. e-mail: henrikhellquist@googlemail.com

*This paper was written by members and invitees of the International Head and Neck Scientific Group (www.IHNSG.com). H.H. and C.A.F. carried out the main part of the paper, and the other authors contributed equally to this study.
} 
11 months. Laryngeal NMC may be underdiagnosed, and an increased awareness among pathologists is warranted. NMC has characteristic morphological features, and positive immunostaining with the NUT antibody is diagnostic. Its aggressive behaviour demands a very intense treatment strategy and the need for its recognition is emphasized further by new promising treatment strategies.

Keywords: BRD4, larynx, NUT, NUT midline carcinoma, t(15;19), undifferentiated carcinoma

\section{Introduction}

Nuclear protein in testis (NUT) midline carcinoma (NMC) is a rare aggressive cancer of which exceedingly few laryngeal cases have been reported. Although NMC is an undifferentiated carcinoma it almost invariably shows features of squamous cell differentiation and is regarded by many as a subtype of squamous cell carcinoma. ${ }^{1-6}$ NMC is characterized by chromosomal rearrangements of the gene encoding NUT at $15 \mathrm{q} 14$, also known as NUTM1 or Chr15orf55. ${ }^{1-7}$ The bromodomain containing 4 (BRD4) gene on $19 \mathrm{q} 13$ is the most common (approximately $70 \%$ of cases) translocation partner gene to NUT resulting in the $\mathrm{t}(15 ; 19)(\mathrm{q} 14 ; \mathrm{p} 13)$ karyotype. ${ }^{8}$ The fusion forms a 6.4-kb fusion oncogene, BRD4-NUT. The encoded oncoprotein BRD4-NUT contributes to carcinogenesis by blocking epithelial cell differentiation and drives growth of the NMC cells. ${ }^{6-9}$ Although recognized only recently, a series of publications since 2001 have established the concept of NUT NMC as a distinct entity. ${ }^{1-25}$

NUT NMC is a poorly differentiated carcinoma that can arise in different organs, but characteristically locates to the midline of head and neck, and mediastinum. Initially NMC was thought to be a childhood cancer, but later studies have shown that it affects people of all ages and there is a slight female predominance of 1.5:1. ${ }^{23}$ The histology ranges from almost entirely undifferentiated carcinomas to carcinomas with focal or even prominent squamous differentiation, and NMC is probably best regarded as a distinctly aggressive subtype of SCC that has a unique and defined genetic abnormality. By 2011 at least 28 cases of NUT NMCs had been reported. Fourteen of the 28 cases had originated in the head and neck, but only one of these in the larynx. ${ }^{18}$ A recent study, however, reported a fivefold increase in the diagnosis of head and neck NMC from 2011 to 2014, and as of 31 December 2014, the International NUT Midline Carcinoma Registry had 48 patients treated for NMC. ${ }^{23}$ Part of this increase is due very probably to the availability of the new antibody specific for the NUT protein, ${ }^{16}$ facilitating more widespread laboratory diagnoses. NUT protein expression is normally exclusive to the testes, and its expression outside the testes is diagnostic of NMC. ${ }^{16}$ The diagnosis of NMC is made by demonstration of the NUT rearrangement either by NUT immunohistochemistry (IHC), fluorescence in-situ hybridization (FISH) or reverse transcription-polymerase chain reaction (RT-PCR) (or cytogenetic analysis). In this study we describe the clinicopathological features of seven cases of laryngeal NMC, the largest series to date, and emphasize the importance of its recognition by the pathologist, particularly as the patients need extremely intensive treatment and also because new promising treatment strategies are at the horizon.

\section{Material and Methods}

Due to its rarity, we made a thorough search of the English language literature (Medline, Pubmed) for any documented laryngeal NMC. We were aware of three unpublished cases from the different hospitals of the collaborating authors, but we also searched for cases in the files of three other different hospital departments by Snomed code T24000 and M codes 80203 (undifferentiated), 80103 [not otherwise specified (NOS)] and 80003 (malignancy NOS). Furthermore, in an attempt to obtain a very crude understanding of the prevalence of laryngeal NMC among laryngeal SCCs, the database (1996-2016) of another hospital was searched. Here all cases of diagnosed laryngeal SCC (M80703) were recorded (of which 100 random cases were reviewed histologically), and during the same time-period all laryngeal cases M coded 80203, 80103 and 80003 were collected (all of which were reviewed histologically).

Immunohistochemistry was performed in five cases using the NUT (C52B1) rabbit monoclonal antibody (mAb \#3625) (Cell Signaling Technology, Inc., Boston, MA, USA). The immunohistochemical procedure was performed strictly according the manufacturer's recommendation for paraffin-embedded tissue. This antibody detects endogenous levels of total NUT protein and endogenous levels of the BRD4-NUT fusion protein found in NUT NMC. In all four laboratories human testis was used as positive control (post-meiotic 
spermatids express NUT at approximately the same level as NMC). In case 7 we also used a case of thymic NUT NMC, established previously by FISH and C52B1. The C52B1 antibody has been shown to be $100 \%$ specific and $87 \%$ sensitive for the diagnosis of NMC, and thus a positive NUT immunostaining by itself is diagnostic of NMC. ${ }^{16,26}$

FISH analysis was performed in four cases. Dualcolour bring-together FISH assays for BRD4 and NUT gene loci using homebrew probes were performed on formalin-fixed, paraffin-embedded, 4- $\mu \mathrm{m}$ tissue sections, as described previously. ${ }^{7}$

\section{Results}

We found only three cases published in the English language literature, ${ }^{13,15,25}$ and have added four new cases of our own. There were four males and three females varying in age from 5 to 78 years, with a mean of 34 years. All seven cases were located in supraglottis and hence none in the glottic or subglottic region. Five patients died of the disease after 3, 7, 8,9 and 11 months. One patient is currently undergoing treatment and survival data were not given in one of the previously published cases. The initial histological diagnosis in two cases was poorly differentiated carcinoma, and the remaining cases undifferentiated carcinoma, invasive basaloid squamous cell carcinoma, high-grade malignant neoplasm, and in two cases NUT carcinoma (Table 1). The diagnosis of NUT NMC in the three previously published cases was based on FISH in two cases and IHC in one. In the three new cases known to us and initiating this study (cases 4-6), the diagnosis was based on IHC (and supplemented by FISH in two of the cases). The search for tumours M coded 80203 . 80103 and 80003 in three other hospitals yielded 38 cases, seven of which had a morphology that could be compatible with NUT NMC. All 38 cases were stained with the C52B1 antibody, and in one of the cases (one of the seven with NUT-compatible morphology) the immunostaining was unequivocally positive (case 7; Table 1). IHC with the NUT antibody was thus positive, with diffuse nuclear staining in five of the cases (Figure 1B). In two cases (cases 2 and 5), FISH showed a reciprocal fusion at both NUT and BRD4 (Figure 1C). The tumour cells were pancytokeratin-, p63- and p40-positive but negative for neuroendocrine, lymphocytic and melanocytic markers.

The retrospective search in one hospital (19962016) yielded seven cases of undifferentiated laryngeal carcinoma compared to the approximately 400 cases of conventional SCC (none of the random 100 cases reviewed histologically showed any undifferentiated areas). All seven patients with undifferentiated carcinoma were deceased (at an age between 35 and 80 years), but we were unable to trace the cause of death. For different reasons, no suitable tissue was available in for IHC in any of the seven cases, thus we could not demonstrate the existence of a single case of laryngeal NUT NMC during the same time that we saw 400 cases of conventional laryngeal squamous cell carcinoma. This is in accordance with previous observations where none of 438 head and neck squamous cell carcinomas stained positively with the C52B1 antibody, ${ }^{16}$ and emphasizes the rarity of NUT NMC, both in the larynx as well as in the head and neck as a whole.

In all cases the histology showed features of an undifferentiated carcinoma consisting of monomorphic cells with round to oval nuclei and distinct nucleoli. The cells were primarily medium-sized and arranged in sheets of different sizes, and cells slightly separated from each other by clear spaces. Many of these relatively discohesive cells had an epithelioid appearance. Typically, the tumours showed abrupt squamous differentiation (Figures 1A,D). Necrosis and high mitotic rate were other features of these tumours.

\section{Discussion}

NMC is defined by rearrangement of the NUT gene. The $\mathrm{t}(15: 19)$ translocation is the most common rearrangement that places NUT in frame with $B R D 4$, the latter being a ubiquitously expressed transcriptional activator that drives expression of the BRD4-NUT fusion gene. The BRD4-NUT fusion was first described in 2003 by French and co-workers. ${ }^{7}$ It has been hypothesized that NMC utilizes a genetic shortcut to the development of SCC, which normally takes years and the accumulation of multiple mutational hits to develop. ${ }^{1,27,28}$ BRD4-NUT forms enormous contiguous stretches of active chromatin (0.1$1.5 \mathrm{Mb})$ that have been termed 'megadomains', enriched with BRD4-NUT and p300. Expression of at least a subset of megadomain-containing genes is critical to the growth of the host NMC cell. For example, the ability of BRD4-NUT to fill whole regulatory compartments for genes such as MYC is thought to be one explanation of the extremely aggressive nature of NMC. ${ }^{6,29}$ In support of this idea, it has been shown that MYC is a key downstream target of BRD4-NUT, and is necessary and sufficient for blockade of 


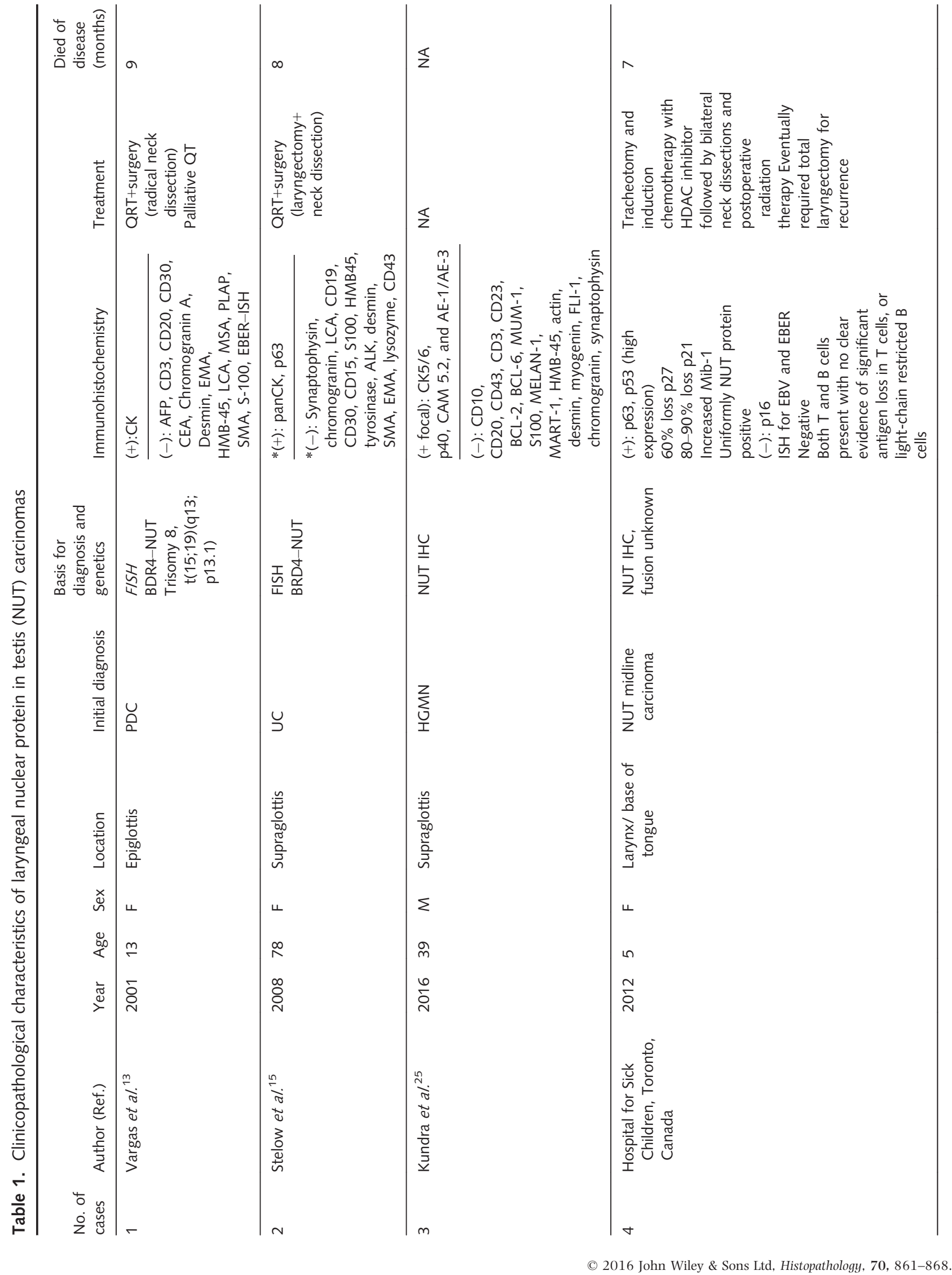




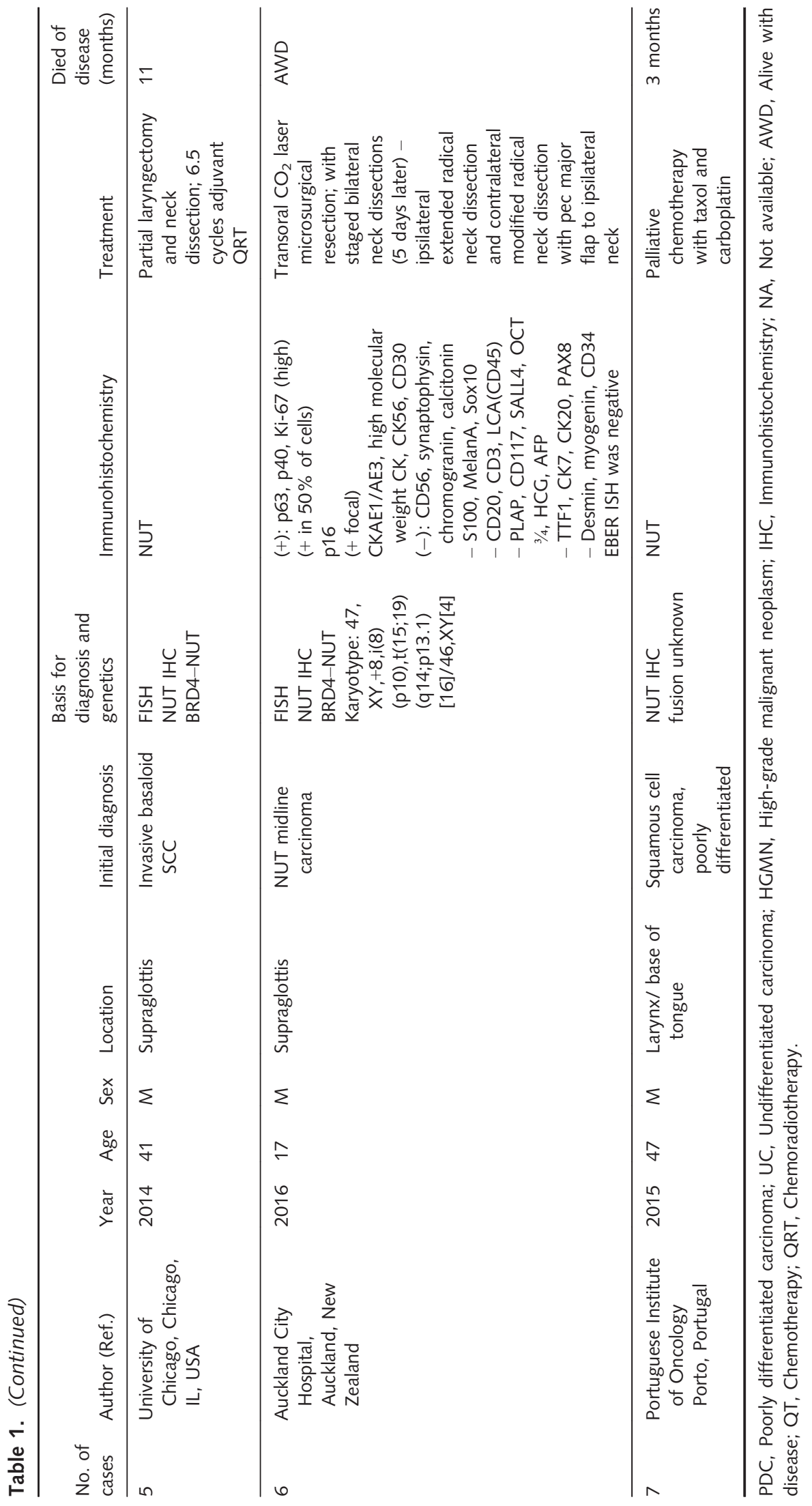



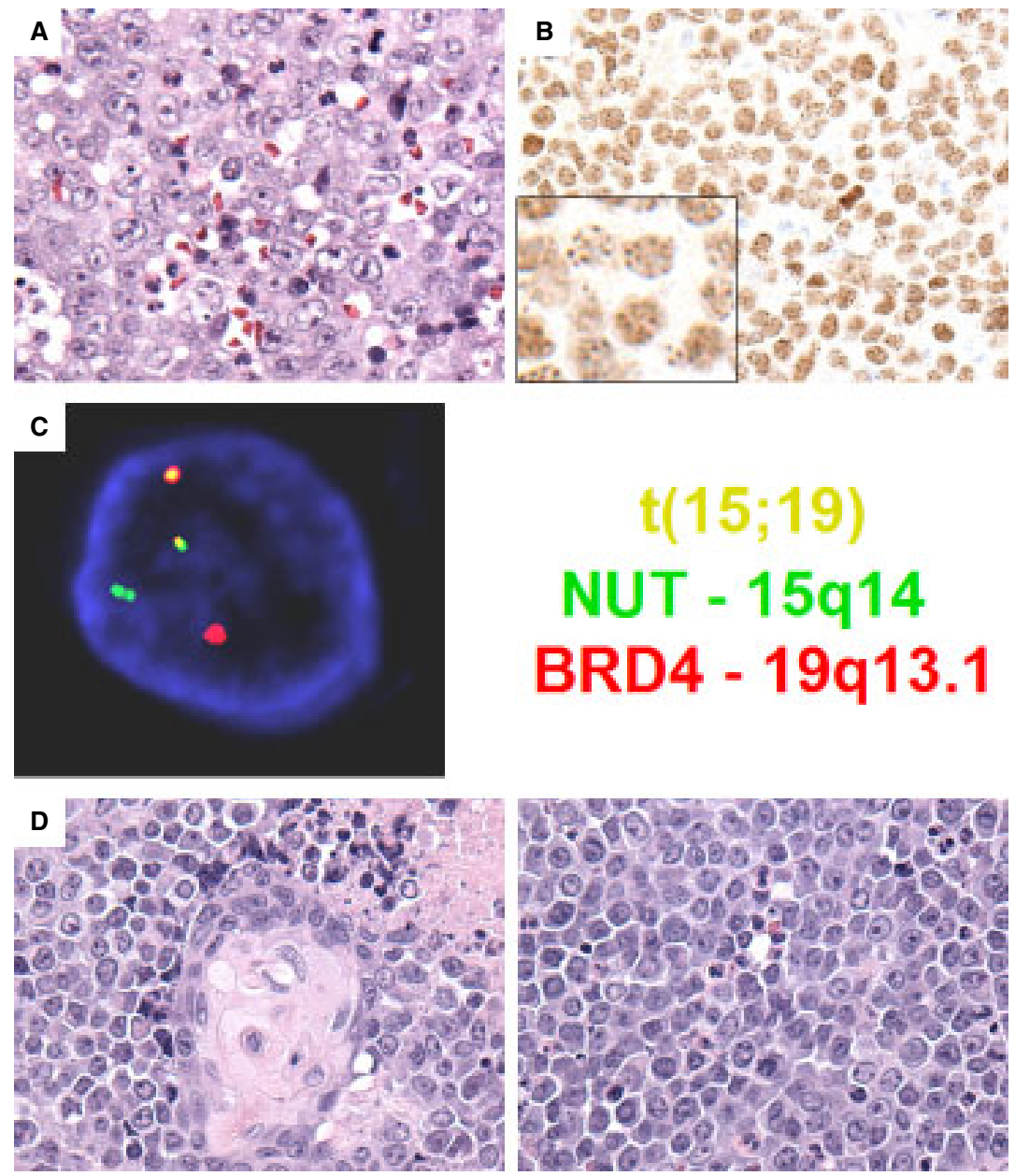

Figure 1. Pathological and molecular features of laryngeal nuclear protein in testis (NUT) midline carcinoma (NMC). A, Case 1 reveals typical undifferentiated morphology of NMC, exhibiting sheets of moderate-sized cells with monomorphic round nuclei and distinct nucleoli. The neutrophilic infiltrate observed in this case is unusual in carcinoma, but seen frequently in NMC (haematoxylin and eosin). B, Diagnostic NUT immunohistochemistry in case 5 revealing diffuse nuclear staining in a speckled pattern (inset). C, Bromodomain containing 4 (BRD4)-NUT fusion fluorescence in-situ hybridization (FISH) using probes covering NUT (green) and BRD4 (red) genes reveals a reciprocal fusion at both NUT (ch. 15q14) and BRD4 (ch. 19p13.1) loci (yellow, overlapping probes). The findings are consistent with a BRD4-NUT fusion in this tumour. D, Case 5 reveals classic NMC morphology with abrupt squamous differentiation (left) and sheets of monomorphic rounds cells separated by clear spaces (right). The frequent single necrotic cells and mitoses are indicative of the aggressive biology of this cancer.

differentiation in NUT carcinomas. ${ }^{3}$ It is of further interest that TP63, a p53 family member whose oncogenic $\Delta \mathrm{N}$ isoform is expressed in the majority of NMCs, maps to a megadomain in all NMC cells tested $^{6}$ and is required for NMC cell viability in vitro. ${ }^{19,30}$

In approximately $75 \%$ of NMCs NUT is fused to $B R D 4, B R D 3$ or $\mathrm{NSD}^{2,5,7,9}$ but in the remaining cases to an unknown partner gene; these latter tumours are termed NUT variants. The commercially available monoclonal antibody C52B1 will detect all forms of NMC, including BRD4-NUT, BRD3-NUT,
NSD3-NUT and NUT variants. ${ }^{17}$ Recently a case of hypopharyngeal NMC was reported that had a novel three-way translocation $\mathrm{t}(9 ; 15 ; 19 ; \mathrm{q} 34 ; \mathrm{q} 13 ; \mathrm{p} 13.1){ }^{20}$ Five of the six cases presented here were positive for the NUT antibody, while the BRD4-NUT fusion was demonstrated by FISH in one case. Our extensive review shows that primary laryngeal NMC is a rarity, and has been underdiagnosed. It shares the same morphology of NMC located elsewhere, and in any case of undifferentiated laryngeal carcinoma IHC with the NUT antibody should be considered. Morphological clues to NMC are features of an undifferentiated 
carcinoma admixed with squamous differentiation, and where the undifferentiated cells tend to be round and may mimic a small round blue cell tumour. The important differential diagnosis of a small round cell tumour has been pointed out by Bishop and associates. $^{30,31}$ Necrosis, including frequent single cell necrosis and high mitotic rate, are present in NMCs. Neutrophilic infiltrates are often present, a feature not seen commonly in other laryngeal carcinomas. A young age of the patient should also alert the pathologist. In our review the mean age was 34 years, which is considerably younger and atypical for conventional laryngeal carcinomas. The patients also tend to present at an advanced clinical stage, often stage IV, illustrated for example by case 7 , where the patient at diagnosis was staged T4aN3M1.

The clinical outcome for patients with laryngeal $\mathrm{NMC}$ and for NMC located elsewhere is, in general, dismal. Chemotherapy or radiation therapy alone for head and neck NMC has been shown to be inadequate, while aggressive initial resection with or without postoperative chemotherapy or radiation has been reported to carry a significantly enhanced survival in a series of 48 patients with NMC. ${ }^{23}$ Nevertheless, head and neck NMC has a poor prognosis in general, and this is the case with laryngeal NMC. Clinical outcome data were available in six patients in our series of seven patients,, and here the survival ranged from 3 to 11 months. A very recent report has shown very promising clinical response by the use of bromodomain and extra-terminal (BET) bromodomain (BRD) inhibitors. The antitumour activity of novel oral BET inhibitor (OTX015/MK-8628) was evaluated in four patients with advanced-stage NMC and showed impressive and rapid antitumour activity in NMC. Two of the patients responded rapidly with tumour regression and a third had meaningful disease stabilization. ${ }^{32}$

We conclude that laryngeal NMC is a rarity, but may be underdiagnosed. It has characteristic morphological features, and positive immunohistochemical staining with the NUT antibody is diagnostic. An increased awareness among pathologists is warranted due to its aggressive behaviour demanding a different treatment strategy than conventional poorly differentiated laryngeal carcinomas.

\section{Acknowledgements}

This work was supported by a grant from the National Institutes of Health, number 2R01CA1246 33 to C.A.F.

\section{Conflicts of interest}

No conflicts of interest to declare.

\section{References}

1. French CA. Pathogenesis of NUT midline carcinoma. Аnnu. Rev. Pathol. 2012; 7; 247-265.

2. Bauer DE, Mitchell CM, Strait KM et al. Clinicopathologic features and long-term outcomes of NUT midline carcinoma. Clin. Cancer Res. 2012; 18; 5773-5779.

3. Grayson AR, Walsh EM, Cameron MJ et al. MYC, a downstream target of BRD-NUT, is necessary and sufficient for the blockade of differentiation in NUT midline carcinoma. Oncogene 2014; 33; 1736-1742.

4. French CA. The importance of diagnosing NUT midline carcinoma. Head Neck Pathol. 2013; 7; 11-16.

5. French CA, Rahman S, Walsh EM et al. NSD3-NUT fusion oncoprotein in NUT midline carcinoma: implications for a novel oncogenic mechanism. Cancer Discov. 2014; 4; 928-941.

6. Alekseyenko AA, Walsh EM, Wang $\mathrm{X}$ et al. The oncogenic BRD4-NUT chromatin regulator drives aberrant transcription within large topological domains. Genes Dev. 2015; 29; $1507-$ 1523.

7. French CA, Kutok JL, Faquin WC et al. Midline carcinoma of children and young adults with NT rearrangement. J. Clin. Oncol. 2004; 22; 4135-4139.

8. French CA, Miyoshi I, Kubonishi I et al. BRD4-NUT fusion oncogene: a novel mechanism in aggressive carcinoma. Cancer Res. 2003; 63; 304-307.

9. French CA, Ramirez CL, Kolmakova J et al. BRD-NUT oncoproteins: a family of closely related nuclear proteins that block epithelial differentiation and maintain the growth of carcinoma cells. Oncogene 2008; 27; 2237-2242.

10. Kubonishi I, Takehara N, Iwata J et al. Novel t(15;19)(q15; p13) chromosome abnormality in a thymic carcinoma. Cancer Res. 1991; 51; 3327-3328.

11. Kees UR, Mulcahy MT, Willoughby ML. Intrathoracic carcinoma in an 11-year-old girl showing a translocation $\mathrm{t}(15 ; 19)$. Am. J. Pediatr. Hematol. Oncol. 1991; 13; 469-464.

12. Lee AC, Kwong YI, Fu KH et al. Disseminated mediastinal carcinoma with chromosomal translocation $(15 ; 19)$. A distinctive clinicopathologic syndrome. Cancer 1993; 72; 2273-2276.

13. Vargas SO, French CA, Faul PN et al. Upper respiratory tract carcinoma with chromosomal translocation 15;19. Evidence for a distinct disease entity of young patients with a rapidly fatal course. Cancer 2001; 92; 1195-1203.

14. Dang TP, Gazdar AF, Virmani AK et al. Chromosome 19 translocation, overexpression of Notch3, and human lung cancer. J. Natl Cancer Inst. 2000; 92; 1355-1357.

15. Stelow EB, Bellizzi AM, Taneja K et al. NUT rearrangement in undifferentiated carcinomas of the upper aerodigestive tract. Am. J. Surg. Pathol. 2008; 32; 828-834.

16. Haack H, Johnson LA, Fry CJ et al. Diagnosis of NUT midline carcinoma using a NUT-specific monoclonal antibody. Am. J. Surg. Pathol. 2009; 33; 984-991.

17. French CA. NUT midline carcinoma. Cancer Genet. Cytogenet. 2010; 203; 16-20.

18. Stelow EB. A review of NUT midline carcinoma. Head Neck Pathol. 2011; 5; 31-35.

19. Bishop JA, Westra WH. NUT midline carcinomas of the sinonasal tract. Am. J. Surg. Pathol. 2012; 36; 1216-1221. 
20. Mills AF, Lanfranchi M, Wein RO et al. NUT midline carcinoma. A case report with a novel translocation and review of the literature. Head Neck Pathol. 2014; 8; 182-186.

21. Sholl LM, Nishino M, Pokharel S et al. Primary pulmonary NUT midline carcinoma: clinical, radiographic, and pathologic characterizations. J. Thorac. Oncol. 2015; 10; 951-959.

22. Andreasen S, French CA, Josiassen M et al. NUT carcinoma of the sublingual gland. Head Neck Pathol. 2016; 10; 362-366.

23. Chau NG, Hurwitz S, Mitchell CM et al. Intensive treatment and survival outcomes in NUT midline carcinoma of the head and neck. Cancer 2016; 122; 3632-3640.

24. Bellizzi AM, Bruzzi C, French CA et al. The cytologic features of NUT midline carcinoma. Cancer 2009; 117; 508-515.

25. Kundra A, Andrei M, Westra W et al. Nuclear protein in testis midline carcinoma of larynx: an underdiagnosed entity. Head Neck 2016; 38; E2471-E2474.

26. Chirieac LR, French CA, Sholl L et al. WHO (2015) classification of tumours of the lung, pleura, thymus and heart. In Travis WD, Brambilla E, Burke AP, Marx A, Nicholson AG eds. Lung: other and unclassified carcinomas. 4th ed. Lyon: IARC, 2015; 97-98.
27. Stransky N, Egloff AM, Tward AD et al. The mutational landscape of head and neck squamous cell carcinoma. Science 2011; 333; 1157-1160.

28. Pickering CR, Zhou JH, Lee JJ et al. Mutational landscape of aggressive cutaneous squamous cell carcinoma. Clin. Cancer Res. 2014; 20; 6582-6592.

29. Reynoird N, Schwartz BE, Delvecchio M et al. Oncogenesis by sequestration of $\mathrm{CBP} / \mathrm{p} 300$ in transcriptionally inactive hyperacetylated chromatin domains. EMBO J. 2010; 29; 29432952.

30. Tilson MP, Bishop JA. Utility of p40 in the differential diagnosis of small round blue cell tumors of the sinonasal tract. Head Neck Pathol. 2014; 8; 141-145.

31. Bishop JA, French CA, Ali SZ. Cytopathological features of NUT midline carcinoma: a series of 26 specimens from 13 patients. Cancer Cytopathol. 2016; 124; 901-908.

32. Stathis A, Zucca E, Bekradda M et al. Clinical response of carcinomas harbouring the BRD4-NUT oncoprotein to the targeted Bromodomain inhibitor OTX015/MK-8628. Cancer Discov. 2016; 6; 492-500. 